\title{
Formation, Mineralization, and Resorption of Bone in Vitamin D-Deficient Rats
}

\author{
D. Baylink, M. Stauffer, J. Wergedal, and C. Rich \\ From the University of Washington School of Medicine and Veterans \\ Administration Hospital, Seattle, Washington 98108
}

\begin{abstract}
A B S T R A C T Quantitative histologic methods have been devised to measure several processes dealing with formation and mineralization of matrix and bone resorption. In vitamin D-deficient rats, the total osteoblastic matrix formation rate was $20 \%$ less and the total osteoclastic bone resorption rate was $80 \%$ more than in pair-fed control rats. These changes were found to be primarily because of changes in the rates of matrix formation and of bone resorption per unit area of forming or resorbing surfaces rather than to changes in the areas of these surfaces. The rate of maturation of osteoid and the rate of initial mineralization both were reduced to half of normal in the vitamin D-deficient rats. These variables related to matrix formation and mineralization were significantly correlated with the concentration of calcium but not with the concentration of phosphate in serum. The occurrence of hypocalcemia is interpreted as the consequence, both of reduced calcium absorption and of inadequate resorptive response of bone cells to homeostatic stimuli, such that, although bone resorption was greater than normal, it did not adequately compensate for the reduced intestinal absorption.
\end{abstract}

\section{INTRODUCTION}

Altered rates of bone formation and resorption in vitamin D-deficient human beings and experimental animals have been reported by numerous investigators. However, evidence has been presented which is consistent with increased bone formation (1), decreased bone formation (2), increased bone resorption (3), and decreased bone resorption (4). Thus, there is no agreement on the effects of vitamin D deficiency on bone formation and resorption. One cause of the difficulty in determining these effects may lie in the inadequately specific methods that have been available to evaluate

Received for publication 2 September 1969 and in revised form 3 February 1970. the complex processes that are altered in vitamin $\mathrm{D}$ deficiency. For example, bone formation, as estimated by kinetic methods, could include mineralization of recently formed matrix, remineralization of areas in mature bone which had become partially demineralized, and deposition along the borders of osteocyte lacunae and canaliculi, while resorption could include both osteoclastic and osteocytic resorption.

The objectives of this study were to measure the effects of vitamin $\mathrm{D}$ deficiency on specific processes associated with bone formation, mineralization, and resorption. To accomplish this, we developed quantitative histological methods to measure directly a number of specific processes in transverse sections taken from the mid-portion of the diaphysis of the tibia of the rat. These, in the volume of bone examined, are the areas of periosteal and endosteal surfaces at which bone formation or resorption occur, the volume of bone formed or resorbed per unit of time per unit area of bone surface involved in active formation or resorption, the total rates of bone formation and resorption and two stages in the course of mineralization of bone matrix, which we have termed the osteoid maturation rate, and the rate of initial mineralization.

\section{METHODS}

The experimental protocol is shown in Fig. 1. All rats were individually housed in the dark in suspended wire cages and fed a semisynthetic diet containing $0.6 \%$ calcium and $0.6 \%$ phosphorus, as described elsewhere (5). 60 male 22 day old Sprague-Dawley rats were divided into three groups, each having a mean weight of about $40 \mathrm{~g}$. The experimental group was given a diet which contained no vitamin $\mathrm{D}$. The control groups were given an identical diet except that it contained 2 IU vitamin $D_{2}$ per $g$. One control group was fed an unrestricted amount of this diet throughout the experimental period. Since preliminary studies showed that when rats were fed a vitamin D-deficient diet weight gain and food consumption were decreased beginning about 10 days after the diet was begun, the other control group was pair fed with the experimental group, starting on the 8th day of the 
experimental period. At this time the amount of vitamin $D_{2}$ in the diet of the pair-fed control group was increased to 3 $\mathrm{IU} / \mathrm{g}$ so that the total consumption of vitamin $\mathrm{D}$ would be comparable in the two control groups.

On the 11th day, each group was further subdivided into two subgroups of 10 rats, each pair of subgroups having the same mean weight. One of each pair of subgroups was sacrificed on the 11th day and the other on the 21st day of the experimental period. The animals sacrificed on the 11th day constituted the basal groups and those on the 21st day the final groups for the purposes of the measurements described below. All of the rats in the basal groups were given $20 \mathrm{mg} / \mathrm{kg}$ body weight of tetracycline ${ }^{1}$ intraperitoneally $6 \mathrm{hr}$ before sacrifice. At the same time that the rats in the basal groups were sacrificed, the rats in the final groups were started on daily intraperitoneal injections of tetracycline which were continued until sacrifice on the 21st day of the experimental period. The dose of tetracycline was $10 \mathrm{mg} / \mathrm{kg}$ body weight except for the first and last injections, which were $20 \mathrm{mg} / \mathrm{kg}$. Blood was obtained from all animals by cardiac puncture just before sacrifice. Serum calcium was measured by atomic absorption spectrophotometry (6) and serum phosphorus by use of an AutoAnalyzer (7).

Each tibia was divided at right angles to its long axis at the fibular junction. A Gillings-Hamco thin-sectioning machine was used to saw three consecutive transverse sections, about $50 \mu$ thick, from the proximal segment of the left tibia. The bone specimen holder was mounted on a goniometer to facilitate sawing sections which were perpendicular to the long axis of the diaphysis. The sawed sections were then hand-ground to a final thickness of about $30 \mu$.

All of the variables measured except the width of the bone area labeled $6 \mathrm{hr}$ after an injection of tetracycline are illustrated schematically in Fig. 2. The most distal two ground sections were mounted unstained in Abopon, ${ }^{2}$ a water-soluble mounting medium, and used for measurements of area, width, and surface length. The entire area of each of the two unstained sections was photographed by use of tungsten and ultraviolet illumination. Enlarged prints with a final magnification of $73 \times$ were then made from each photomicrograph. Measurements of area were made on the enlarged prints with a planimeter and surface lengths were measured by means of a map meter. A bone-forming surface was identified by the presence of a mineralization front or an appositional tetracycline label or a band of osteoid. All surfaces along which formation was not active were classified as resorbing surfaces; the validity of this criterion was evaluated in a separate experiment described later. Osteoid was not included in any measurements of area and surface length made on photographic prints. The most proximal ground section was stained with a modified von Kossa procedure (8), counterstained with nuclear fast red (9), dehydrated in acetone, cleared in xylene, and mounted in Fluormount ${ }^{3}$ for measurements of osteoid width and, in the basal groups, for measurements of the length of formation and resorption surfaces. The line sampling method was used (Zeiss No. II type integrating eyepiece). A filar micrometer was used to measure osteoid width. The von Kossa procedure stained the mineralization front black with metallic silver and nuclear fast red stained the nuclei of osteoblasts red and osteoid yellow to orange. Because nuclear fast red is

${ }^{1}$ Demethylchlortetracycline, kindly supplied by Dr. Hugh Macdonald, Lederle Laboratories, Pearl River, N. Y.

${ }^{2}$ Valnor Corporation, Brooklyn, N. Y.

${ }^{3}$ E. Gurr Ltd., London, S. W. 14, England.
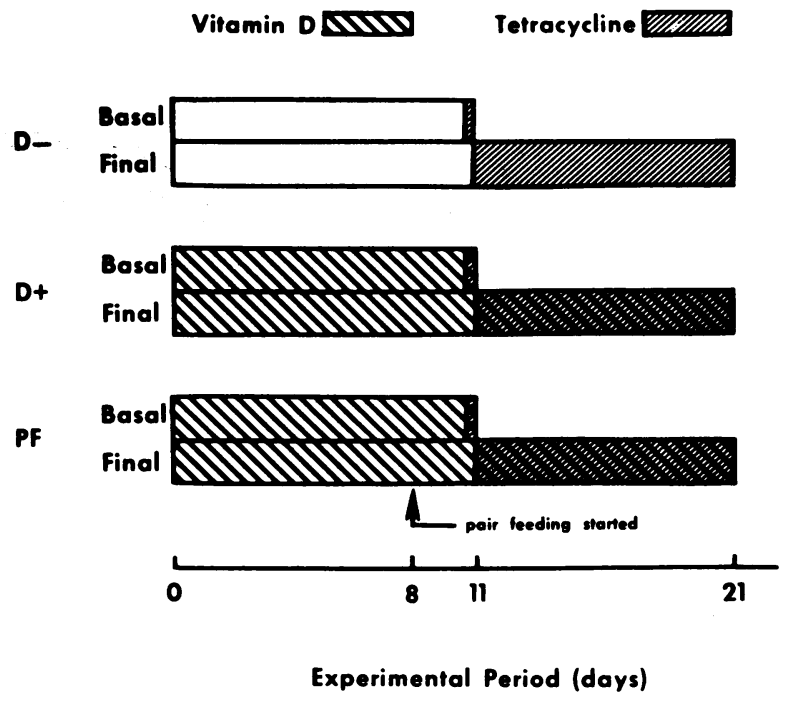

Figure 1 Experimental Protocol. Vitamin D-deficient groups (D-) received no vitamin $D$ in the diet. The ad lib.-fed control groups (D+) received a diet containing 2 IU of vitamin $D_{2}$ per $g$. The pair-fed control group received the same diet until the 8th day when pair feeding was started and thereafter $3 \mathrm{IU}$ of vitamin $\mathrm{D}_{2}$ per $\mathrm{g}$.

fluorescent, the sections were viewed with a combination of tungsten and ultraviolet illumination. In contrast to our results with other staining methods, this method allowed us to distinguish precisely the junction between osteoid and the mineralization front (Fig. 2). Since the width of osteoid was not constant around the periosteum, osteoid width was measured every $60^{\circ}$ around the periosteal circumference with the aid of a protractor placed on top of the slide. In a preliminary experiment, we found that the mean osteoid width obtained by this procedure did not differ by more than $3 \%$ of the mean of measurements made every $10^{\circ}$ around the periosteal circumference. The endosteal osteoid width was measured at four equidistant positions along the endosteal osteoid border. The width of bone labeled $6 \mathrm{hr}$ after an injection of tetracycline was measured in unstained ground sections from all rats of the basal groups, by means of ultraviolet illumination and the same sampling procedure as was described above for measurement of osteoid width.

Although no shrinkage of mineralized bone was found when unstained ground sections mounted in water were compared with the same sections mounted in Abopon, it was apparent that osteoid shrank during dehydration. Because nuclear fast red is soluble in water, dehydration was necessary in order to mount sections using this stain. Therefore, ground sections stained with nuclear fast red and the von Kossa procedure were measured for osteoid width immediately after being mounted in water and then again after they had been dehydrated in acetone, cleared in xylene, and mounted in Fluormount. The osteoid width, measured as described above in six sections, was found to decrease, as a result of dehydration, by $26.7 \pm 3.1 \%$ in control rats and $24.4 \pm 2.6 \%$ in vitamin $D$-deficient rats. The difference between these two values was not statistically significant and, therefore, the combined mean value of $25.5 \%$ was applied

*These and all other results are given as the mean \pm SD. 


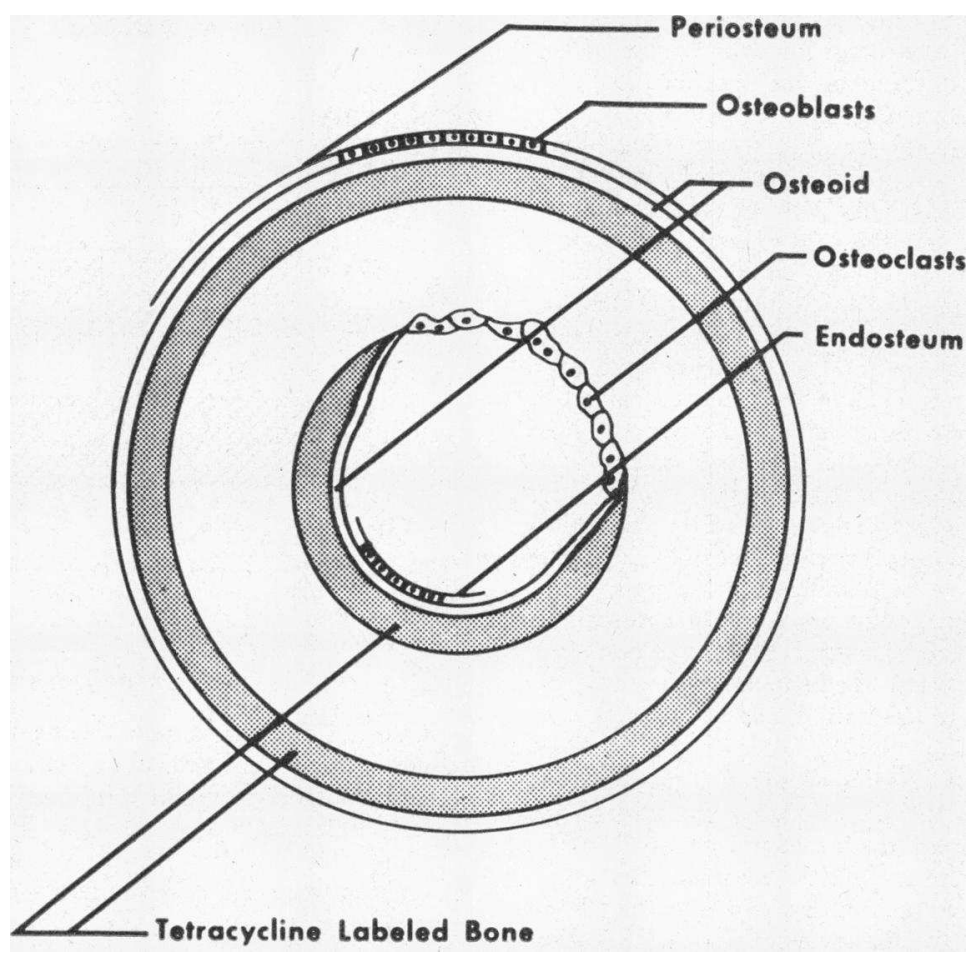

Figure 2 Schema of a transverse section of tibia from a rat given tetracycline for 10 days.

to correct all of the measured osteoid for shrinkage so that osteoid and bone measurements would correspond and apply to the hydrated state.

The mineralization front is defined as the amount of newly mineralized bone (adjacent to osteoid) into which tetracycline diffuses $(10,11)$. The maximum concentration of mineral within the mineralization front was estimated from refractive index (nD) measurements. It has been shown that there is a linear proportionality between the concentration of hydroxyapatite in bone and its refractive index and that this relationship holds throughout the entire range from unmineralized bone matrix to maximally mineralized bone (12). The $\mathrm{nD}$ of osteoid, the maximum $\mathrm{nD}$ in the mineralization front, and the maximum $\mathrm{nD}$ in bone formed before the experimental period were measured at the edge of a bisected transverse ground section by means of the Becke line procedure, in which monochromatic light $(5460 \mathrm{~A})$ and a series of oils with $\mathrm{nD}$ gradations of $0.002^{5}$ were used (13).

Calculations. The precision of the methods used to make measurements of width, area, and surface are given in Table I. Except when noted otherwise the units are cubic millimeters for volume, square millimeters for area, millimeters for length and width, and days for time. The differences in the measurements between the three sections of bone examined were not statistically significant. Therefore, each section was considered to be representative of the entire site and, when the same variable was measured on more than one section from an individual rat, the mean value was used. Since the length of the segment of the tibial shaft from which the three sections were taken was more

\footnotetext{
${ }^{5}$ R. P. Cargill Labs., Inc., Cedar Grove, N. J.
}

than $1 \mathrm{~mm}$, the measured rates could be expressed as the volumes of bone formed and resorbed per day.

The area occupied by osteoid and that labeled $6 \mathrm{hr}$ after an injection of tetracycline were calculated from measurements of width and circumference, using formulas for the area of an annulus,

$$
\mathrm{W}\left(L_{\mathrm{i}}+\pi \mathrm{W}\right)=\mathrm{A}=\mathrm{W}\left(\mathrm{L}_{0}-\pi \mathrm{W}\right),
$$

or for the area of a segment of an annulus,

$$
\frac{W L_{i}}{L_{i}}\left(L_{i}+\pi W\right)=A=\frac{W L_{f}}{L_{0}}\left(L_{0}-\pi W\right),
$$

where $A$ is area, $W$ is width and $L_{1}$ and $L_{0}$ are the internal and external circumferences, respectively, and $L_{q}$ is the length of endosteal matrix forming surface. The accuracy of this approximation was tested in the control and experimental groups at the end of the experimental period by comparing the total bone area, calculated on the basis of an assumption that the periosteal circumference is a circle, with the measured total area. The area so calculated was found to be $3 \%$ more than the measured area.

The derived variables are described and the formulas used to calculate them are given below.

(a) Total bone formation rate $\left(R_{t b p}\right)$. This is a measurement of the rate of formation of mineralized bone. All bone formed during the 10 day measuring period is labeled with tetracycline. In addition, tetracycline deposits in the mineralization front of bone formed before the start of the measuring period. Therefore, to determine the true amount of bone formed, the area labeled during the first $6 \mathrm{hr}$ (which consists of the mineralization front plus bone mineralized during the $6 \mathrm{hr}$ period) is subtracted from the total amount 
TABLE I

The Results of Measurements of Width, Area, and Surface, the Methods Used to Measure Them, and the Precision of the Methods

\begin{tabular}{|c|c|c|c|c|c|}
\hline \multirow[b]{2}{*}{ Measurement* } & \multirow[b]{2}{*}{$\begin{array}{l}\text { Ad lib.- } \\
\text { fed control }\end{array}$} & \multicolumn{2}{|c|}{ Results } & \multirow[b]{2}{*}{ Method } & \multirow[b]{2}{*}{ Precisiont } \\
\hline & & $\begin{array}{l}\text { Pair-fed } \\
\text { control }\end{array}$ & $\begin{array}{l}\text { Vitamin D } \\
\text { deficient }\end{array}$ & & \\
\hline Width $(\mu)$ & & & & & $\%$ \\
\hline Basal periosteal osteoid§ & $13.9 \pm 0.7 \|$ & $12.8 \pm 1.5$ & $18.7 \pm 3.7$ & Filar micrometer & \pm 2 \\
\hline Basal endosteal osteoid & $6.8 \pm 2.2$ & $8.1 \pm 1.8$ & $9.5 \pm 2.5$ & " & \pm 4 \\
\hline Basal periosteal $6 \mathrm{hr}$ label & $6.3 \pm 0.7$ & $6.2 \pm 0.6$ & $7.2 \pm 1.7$ & $"$ & \pm 3 \\
\hline Basal endosteal $6 \mathrm{hr}$ label & $2.9 \pm 0.4$ & $3.8 \pm 0.3$ & $4.1 \pm 0.9$ & $"$ & \pm 4 \\
\hline Final periosteal osteoid $\uparrow$ & $11.3 \pm 1.2$ & $12.9 \pm 1.8$ & $21.4 \pm 5.2$ & $"$ & \pm 2 \\
\hline Final endosteal osteoid & $6.1 \pm 0.8$ & $6.6 \pm 1.0$ & $13.6 \pm 4.4$ & $"$ & \pm 4 \\
\hline \multicolumn{6}{|l|}{ Area $\left(m m^{2}\right)$} \\
\hline Basal total** & $2.66 \pm 0.12$ & $2.59 \pm 0.10$ & $2.56 \pm 0.14$ & Planimeter & \pm 1 \\
\hline Basal medullary & $0.98 \pm 0.06$ & $0.96 \pm 0.06$ & $0.91 \pm 0.08$ & , & \pm 1 \\
\hline Final total & $3.17 \pm 0.13$ & $3.08 \pm 0.12$ & $2.97 \pm 0.18$ & $"$ & \pm 1 \\
\hline Initial total $\ddagger$ & $2.52 \pm 0.11$ & $2.48 \pm 0.12$ & $2.53 \pm 0.13$ & $"$ & \pm 1 \\
\hline Final medullary & $0.91 \pm 0.09$ & $0.94 \pm 0.07$ & $0.99 \pm 0.12$ & $"$ & \pm 1 \\
\hline Endosteal labeled & $0.11 \pm 0.02$ & $0.12 \pm 0.03$ & $0.10 \pm 0.02$ & $"$ & \pm 1 \\
\hline \multicolumn{6}{|l|}{ Surface $(m m)$} \\
\hline Basal periosteal & $5.86 \pm 0.13$ & $5.79 \pm 0.13$ & $5.73 \pm 0.16$ & Map-meter & \pm 1 \\
\hline Basal endosteal forming & $1.85 \pm 0.56$ & $1.90 \pm 0.25$ & $1.95 \pm 0.40$ & Line-sampling & \pm 6 \\
\hline Basal endosteal resorbing & $1.93 \pm 0.63$ & $1.78 \pm 0.34$ & $1.65 \pm 0.35$ & $"$ & \pm 6 \\
\hline Final periosteal & $6.41 \pm 0.15$ & $6.29 \pm 0.13$ & $6.21 \pm 0.19$ & Map-meter & \pm 1 \\
\hline Initial periostealłł & $5.69 \pm 0.12$ & $5.64 \pm 0.12$ & $5.67 \pm 0.16$ & $"$ & \pm 1 \\
\hline Final endosteal forming & $2.90 \pm 0.32$ & $2.87 \pm 0.44$ & $2.70 \pm 0.50$ & $"$ & \pm 2 \\
\hline Final endosteal resorbing & $0.61 \pm 0.24$ & $0.71 \pm 0.40$ & $0.93 \pm 0.45$ & $"$ & \pm 2 \\
\hline
\end{tabular}

* See methods section for definition of variables.

$\ddagger$ Coefficient of variation.

$\S$ Measurements made on bone from animals in the basal group.

$\|$ Mean \pm sD.

T Measurements made on bone from animals in the final group.

** Total area is the area circumscribed by the periosteal border of bone and includes the medullary cavity.

$\sharp$ These values were measured in the final group. Since all bone formed during the experimental period is labeled, the location of the initial periosteal border is given by the junction between labeled and unlabeled periosteal bone.

of periosteal and endosteal bone labeled during the measuring period.

$$
\mathrm{R}_{\mathrm{tbf}}=\frac{\mathrm{A}_{\mathrm{pf}}-\mathrm{A}_{\mathrm{pb}}+\mathrm{A}_{\mathrm{ef}}-\overline{\mathrm{A}}_{\mathrm{eb}}}{\mathrm{T}_{\mathrm{f}}-\mathrm{T}_{\mathrm{b}}},
$$

where $A_{p f}$ and $A_{e f}$ are the periosteal and endosteal labeled areas labeled after 10 days of tetracycline in the final groups, $\mathrm{A}_{\mathrm{pb}}$ and $\overline{\mathrm{A}}_{\mathrm{eb}}$ are the labeled areas after $6 \mathrm{hr}$ of tetracycline in the basal groups, and $T_{t}$ and $T_{b}$ are the two labeling periods (10 and 0.25 days, respectively). $A_{p f}$ is the total area enclosed by the periosteal border of tetracycline label minus the area deep to the internal border of this label. $A_{p b}$ is calculated from measurements of periostal circumference and mean $6 \mathrm{hr}$ periosteal label width in the basal group, by means of the formula for the area of an annulus. $\overline{\mathrm{A}}_{\mathrm{eb}}$ is a mean value calculated from the length of endosteal-forming surface and $6 \mathrm{hr}$ endosteal label width, by use of the formula for a sector of an annulus. (b) Total matrix formation rate $\left(R_{t m f}\right)$. This includes all matrix formed, both osteoid and mineralized bone. It is calculated by adding the total amount of bone formed to the increase in the amount of osteoid which occurred during the measuring period and dividing by the length of the measuring period.

$$
\mathrm{R}_{\mathrm{tmf}}=\mathrm{R}_{\mathrm{tbf}}+\frac{\mathrm{A}_{\mathrm{of}}-\mathrm{A}_{\mathrm{ob}}}{\mathrm{T}_{\mathrm{f}}}
$$

where $A_{o r}$ and $A_{o b}$ are the total areas of osteoid in the final and basal groups. These areas are calculated from circumference or segment length and average band width, using the formulas for the area of an annulus or the segment of an annulus, as appropriate.

(c) Periosteal bone apposition rate $\left(R_{p b a}\right)$. This is the width of mineralized bone formed per unit length of boneforming surface and is calculated by dividing the periosteal 
bone formation rate by the average length of bone-forming surface at the periosteum.

$$
R_{p b a}=\frac{A_{p f}-A_{p b}}{T_{f}-T_{b}} \cdot \frac{2}{L_{f}+L_{i}},
$$

where $L_{f}$ is the length of the circumference of the periosteal border of the periosteal label and $L_{1}$ is the length of the deep border of the periosteal label, both measured in the final group ( $L_{1}$ gives the position of the periosteal border at the start of the measuring period).

(d) Periosteal matrix apposition rate $\left(R_{p m a}\right)$. This includes all matrix formed, whether mineralized or not, at the end of the measuring period.

$$
R_{p m a}=R_{p b a}+\frac{W_{o f}-\bar{W}_{o b}}{T_{f}},
$$

where $\mathrm{W}_{\text {of }}$ is the final periosteal osteoid width and $\overline{\mathrm{W}}_{\mathrm{ob}}$ is the mean basal periosteal osteoid width. The endosteal matrix apposition rate was calculated in a similar manner.

(e) Mineralization lag time $\left(T_{m}\right)$. This is the time between the formation of osteoid and the subsequent onset of mineralization in this osteoid. It was calculated at the periosteum and endosteum by dividing the mean osteoid width for the 10 day experimental period by the corresponding matrix apposition rate, by the formula,

$$
T_{m}{ }^{-}=\frac{\bar{W}_{o b}+W_{o f}}{2 R_{m a}},
$$

where $R_{m a}$ is the matrix apposition rate at either the periosteum or endosteum.

(f) Osteoid maturation rate $\left(R_{0}\right)$. This is based on the concept that a sequence of changes occurs in osteoid between when it is formed by osteoblasts and when mineralization starts and that, in respect to these changes, it is $0 \%$ mature when it is formed and $100 \%$ mature when mineralization is initiated. It is expressed in per cent per hour.

$$
R_{0}=\frac{100}{T_{m} \times 24}
$$

(g) Periosteal initial mineralization rate $\left(R_{m}\right)$. This rate is defined as the rate at which the concentration of mineral increases in the periosteal mineralization front. To calculate this, it is necessary to determine the maximum concentration of hydroxyapatite in the mineralization front. This was calculated from refractive index $(\mathrm{nD})$ measurements by the formula,

$$
\mathrm{C}=\frac{\mathrm{nD}_{\mathrm{mf}}-\mathrm{nD}_{0}}{\mathrm{nD}_{\max }-\mathrm{nD}_{0}}
$$

where $\mathrm{C}$ is the maximal mineral concentration in the mineralization front (expressed as a fraction of the maximum mineral concentration in mature bone), $\mathrm{nD}_{\mathrm{ms}}$ is the maximum refractive index in the mineralization front, $\mathrm{nD}_{0}$ is the refractive index in osteoid, and $\mathrm{nD}_{\max }$ is the refractive index in bone formed before the start of the experiment. The time required to achieve this mineral concentration is calculated by dividing the width of the mineralization front by the bone apposition rate. After an intraperitoneal injection of tetracycline, the blood level is sufficiently high to label all bone formed for at least $6 \mathrm{hr}(10)$. Therefore, since in the basal groups, the $6 \mathrm{hr}$ tetracycline label width included the mineralization front plus bone labeled as a result of $6 \mathrm{hr}$ of apposition, the width of the mineralization front was calculated by subtracting the width of bone formed in $6 \mathrm{hr}$ from the total width of bone labeled $6 \mathrm{hr}$ after an injection of tetracycline.

In the final groups, the mineralizing front could not be measured because it could not be distinguished from the remainder of the 10 day label. Since, in the basal groups, there was a significant relationship $(r=0.80, P<0.001)$ between osteoid width and mineralizing front width, this relationship was used to estimate the final mineralizing front width from the measured final osteoid width by the estimating equation,

$$
\mathrm{W}_{\mathrm{pmf}}=0.31 \mathrm{~W}_{\mathrm{po}}-0.00036,
$$

where $\mathrm{W}_{\mathrm{pm}}$ is the final periosteal mineralizing front width and $W_{p o}$ is the final periosteal osteoid width.

The initial mineralization rate at the periosteum was calculated by the formula,

$$
R_{m}=\frac{2 C R_{p b a}}{\bar{W}_{m f b}+W_{m f f}},
$$

where $\overline{\mathrm{W}}_{\mathrm{mpb}}$ is the mean basal mineralization front width and $W_{\text {mes }}$ is the final mineralization front width. The time unit of the rates so calculated was converted from days to hours because $20 \%$ of maximum mineralization was reached in less than 1 day. This rate should not be applied to individual points between 0 and $20 \%$ of maximum mineralization because we have found by means of electron microprobe analyses of calcium and phosphorus concentration that the mineralization rate is not constant between 0 and $20 \%$ of maximum mineralization.

(h) Endosteal bone resorption rate $\left(R_{e b r}\right)$. Since no resorption occurred at the periosteum, this represents the total resorption rate. In principle, the endosteal osteoclastic bone resorption rate was calculated by adding the difference in medullary area between corresponding basal and final groups to the area of endosteal labeled bone and dividing the sum by the length of the experimental period. Since the basal medullary area did not contain the mineralization front, it was necessary to subtract the area of the basal endosteal mineralization front from the endosteal area labeled by tetracycline in the final groups to obtain the true change in medullary area due to resorption. The endosteal bone resorption rate was calculated by the formula,

$$
R_{e b r}=\frac{A_{m f}-A_{m b}+A_{e f}-\bar{A}_{e m f}}{T_{f}},
$$

where $A_{m f}$ is the final medullary area, $A_{m b}$ is the basal medullary area, $A_{e f}$ is the area of endosteal labeled bone in the final group of rats, and $\bar{A}_{e m f}$ is the mean basal endosteal mineralization front area.

(i) The linear rate of endosteal bone resorption $\left(R_{l b r}\right)$. This is the mean width of endosteal bone resorbed per day per unit length of bone resorbing surface and is analogous to the periosteal bone apposition rate. It was calculated by dividing the area of bone resorbed per day by the mean of the basal and final endosteal bone resorbing surfaces by the formula,

$$
\mathrm{R}_{\mathrm{lbr}}=\frac{2 \mathrm{R}_{\mathrm{ebr}}}{\overline{\mathrm{L}}_{\mathrm{rb}}+\mathrm{L}_{\mathrm{rf}}}
$$

where $\bar{L}_{r b}$ is the mean basal endosteal bone resorbing surface and $L_{r f}$ is the final endosteal bone resorbing surface.

Significance estimates were made by means of Student's $t$ test on variables calculated from the formulae given above,

'Baylink, D., J. Wergedal, and M. Stauffer. Unpublished data. 

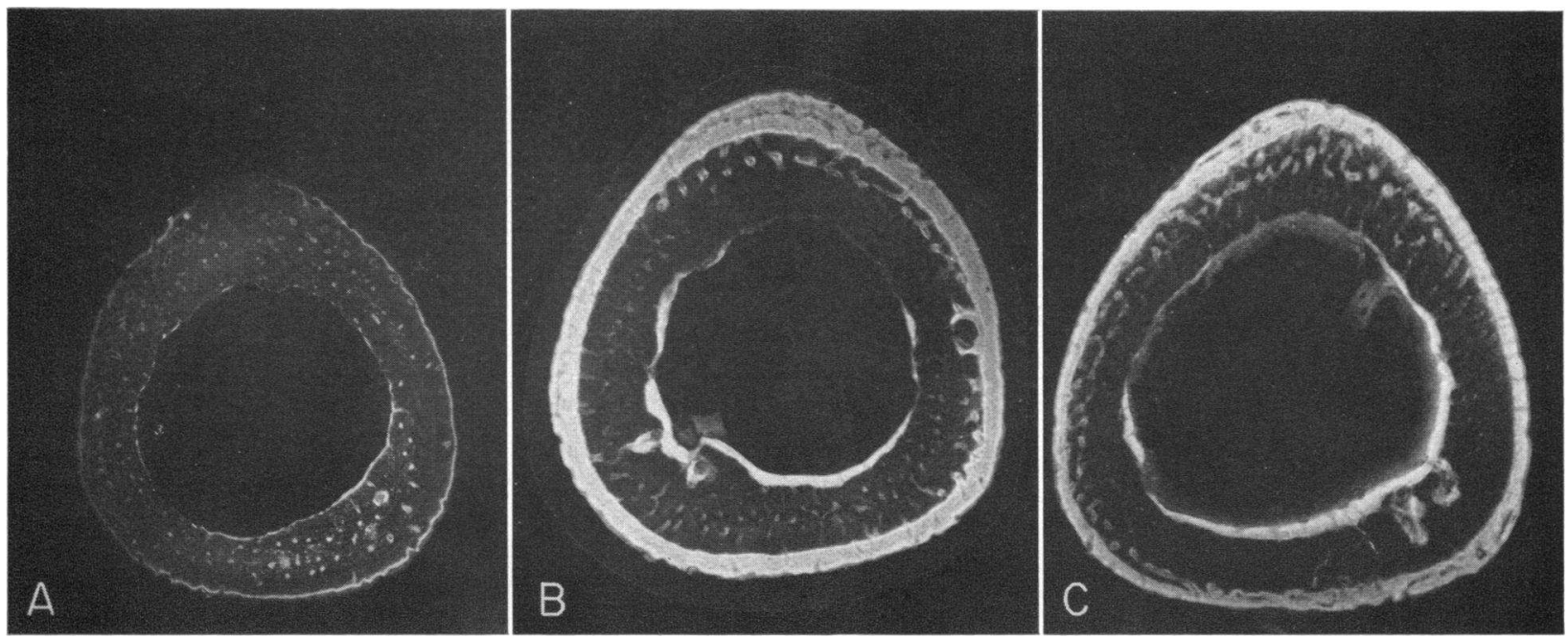

Figure 3 Fluorescence photomicrographs of sections of bone from a pair-fed control rat in the basal group (A) sacrificed at 33 days of age, at the start of the period during which measurements of bone formation and resorption were made, and from pair-fed control (B) and vitamin D-deficient $(C)$ rats sacrificed 10 days later. An intraperitoneal injection of tetracycline had been given to the rat in the basal group $6 \mathrm{hr}$ before sacrifice and can be seen where it is deposited in the mineralization front as a thin band around the entire periosteal perimeter and along a segment of the endosteal perimeter of bone. Tetracycline was given to the control and vitamin D-deficient rats continuously between the 33rd and 43rd days and can be seen as broad periosteal and endosteal bands in bone which was mineralized during that time. Note that bone was being formed along a greater segment of the endosteal surface in 43 than in 33 day old rats. This illustrates that, in this part of the tibia of rats of this age, there was a progressive increase in the extent of the endosteal surface along which bone is formed. Because of this and other reasons, bone formed after the 11th day is not resorbed during the experimental period. $\times 33$.

with the exception of bone resorption. Since it was not technically possible to measure directly the initial medullary area of rats in the final groups, we could not determine the resorption rate for each rat individually, and consequently we could not estimate the true variance for the resorption rate. However, by using analysis of variance we were able to determine a variance which could be used in conjunction with Scheffe's multiple comparison technique (14) for a valid statistical analysis of the difference between the mean resorption rates. Thus, the variance of the bone resorption rate essentially was determined by summing the variance of the basal medullary area and the variance of the sum of the final medullary area and endosteal labeled area. Because each of these two variables is an order of magnitude greater than the total amount of bone resorbed during the measuring period (Tables I and III) and because the variance for bone resorption was considerably greater than any other measurement made in this work, the variance so calculated was undoubtedly much higher than the true variance. Therefore, we estimated variance by a second procedure, which provided individual resorption rates, using the reasonable assumption that rats in the final groups with smaller medullary areas had smaller initial medullary areas than those with larger medullary areas. Thus, we ranked rats in the basal groups according to the sum of the medullary area and rats in the final groups according to the sum of the medullary area and endosteal tetracycline-labeled area in descending order and then, using rats of corresponding rank position, we calculated the difference between these two parameters to obtain individual bone resorption rates.

Validation experiments. To determine whether or not bone labeled during the 10 day experimental period was resorbed before the end of that period, 33 day old pair-fed and vitamin $\mathrm{D}$-deficient rats were given intraperitoneal doses of tetracycline $30 \mathrm{mg} / \mathrm{kg}$ body weight and alizarin red S 25 $\mathrm{mg} / \mathrm{kg}$ body weight alternately every $3 \mathrm{rd}$ day for 12 days. (An ad lib.-fed control group was not included because a preliminary experiment, using our method for measuring resorption and 10 rats per group, showed that the resorption rate in these two groups was greater than in the ad lib.-fed control group.) Two animals from each group were sacrificed $24 \mathrm{hr}$ after each injection and ground sections were prepared from our sampling site in the tibial diaphysis. In these sections the periosteum and endosteum were evaluated for the presence of Howship's lacunae in labeled bone, for the presence of osteoid adjacent to the last label, and for the presence of an alternating label pattern (10).

A separate experiment was carried out on ad lib.-fed control rats of the same age as the basal and final groups at time of sacrifice to determine how much of the unlabeled endosteal surface was involved in active osteoclastic resorption at the time of sacrifice. Tetracycline, $20 \mathrm{mg} / \mathrm{kg}$ body weight, was given intraperitoneally daily for 2 days before sacrifice in order to label that portion of the surface actively involved in bone formation. Immediately after sacrifice, fresh unfixed ground sections were stained for acid phosphatase using the naphthol AS-TR phosphate method (15). We and 


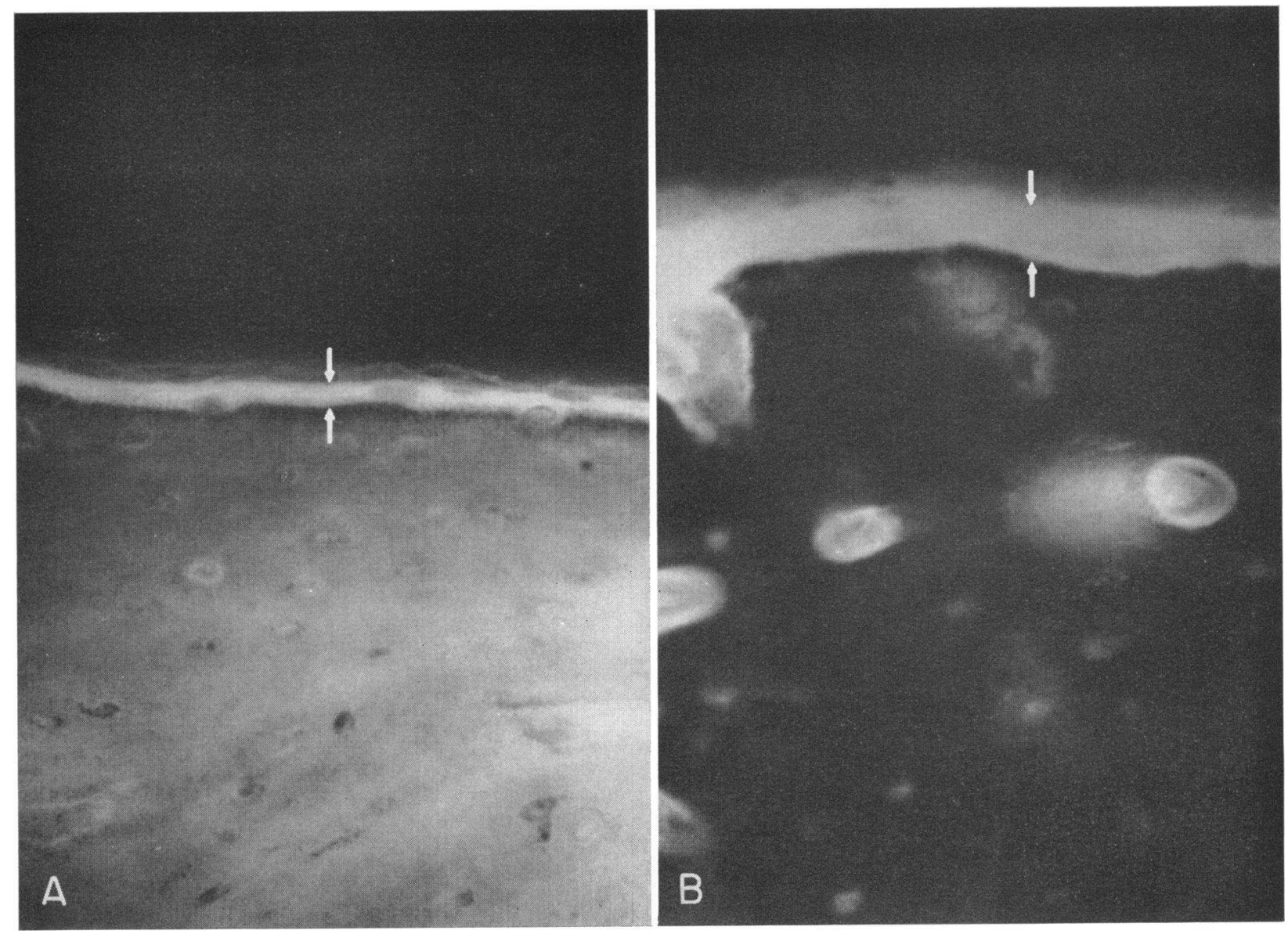

FIGURE 4 Fluorescence photomicrographs of periosteal osteoid on the 11th day of the experimental period from (A) a pair-fed control rat and (B) a vitamin D-deficient rat. The arrows indicate the inner and outer borders of osteoid. The mineralization front is stained with silver and appears black in this photomicrograph. $\times 425$.

others have shown that sites of osteoclastic resorption display a highly characteristic intense extracellular acid phosphatase reaction along the borders of Howship's lacunae as well as intracellular acid phosphatase activity in osteoclasts $(16,17)$. The presence of this reaction along the endosteal bone surface was used to identify sites where osteoclastic resorption was active at the time of sacrifice (16) and the presence of in vivo appositional tetracycline uptake was used to identify sites of active bone formation. Acid phosphatase activity at the endosteal and periosteal bone surfaces was also evaluated in control and vitamin $\mathrm{D}$-deficient rats at numerous time intervals between 33 and 43 days of age.

\section{RESULTS}

A continuous band of osteoid covered by osteoblasts and overlying a continuous band of tetracycline-labeled bone was found around the entire periosteal circumference (Figs. 3 and 4 ). In the validation experiment in which tetracycline and alizarin red $\mathrm{S}$ were given alternately, these labels were always found as alternating continuous bands at the periosteum. Howship's lacunae, osteoclasts, or intense acid phosphatase activity was never found at the periosteum. Thus, there was no evidence for bone resorption at the periosteum during the experimental period.

Bone formation at the endosteum was localized within a specific region (Fig. 3). Along the remainder of the endosteum, the presence of Howship's lacunae, osteoclasts, and a characteristically intense acid phosphatase reaction indicated that osteoclastic bone resorption was active at the time of sampling. Since evidence of endosteal bone resorption was seen in both the basal and final groups, it was necessary to determine that no endosteal bone formed and labeled during the 10 day measuring period was resorbed before the end of this period. When rats were injected with tetracycline and alizarin red $S$ alternately, the yellow tetracycline label was always found adjacent to a red alizarin red $\mathrm{S}$ label 


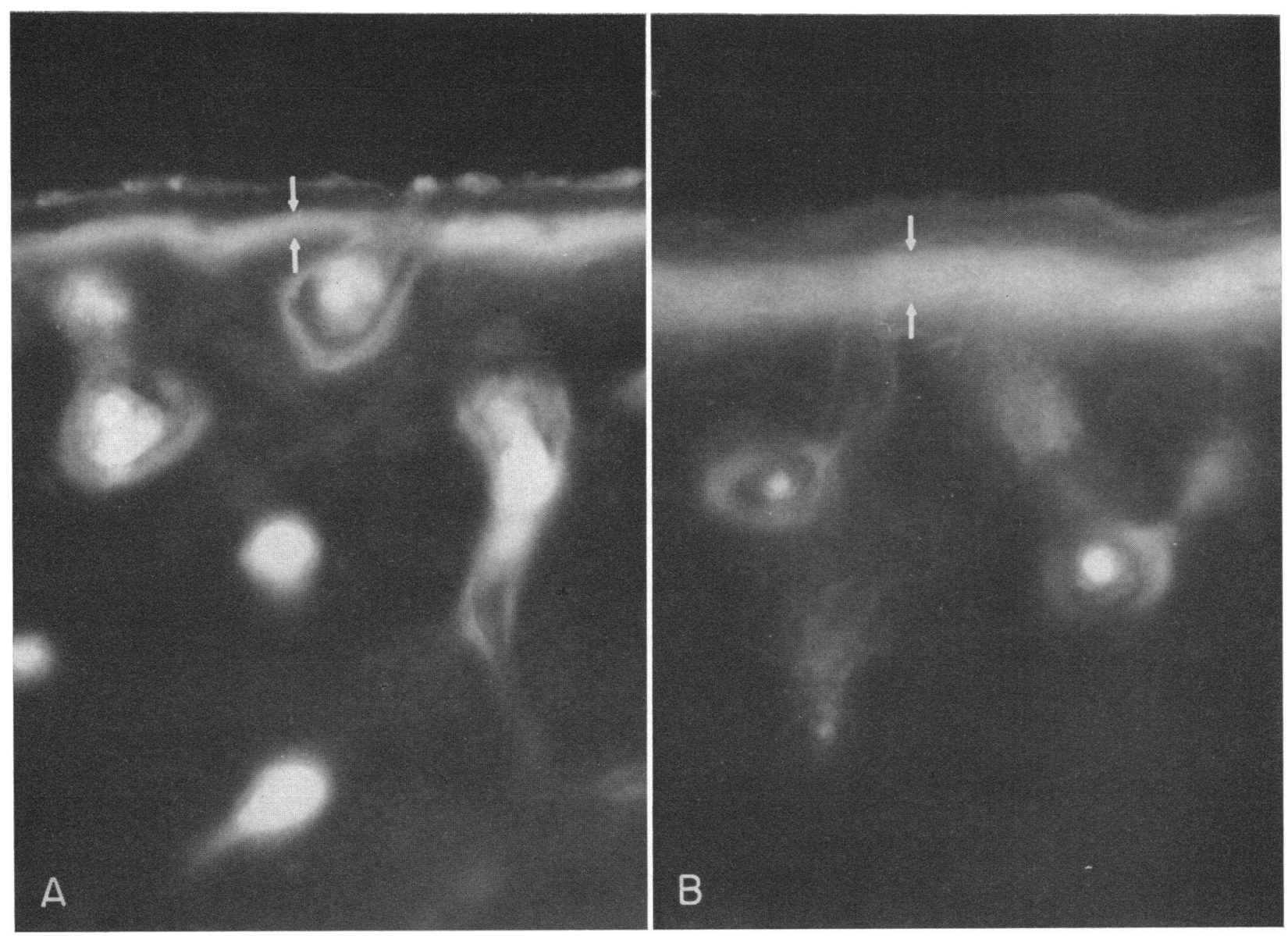

FIGURE 5 Fluorescence photomicrographs of the area labeled $6 \mathrm{hr}$ after an i.p. injection of tetracycline on the 11th day of the experimental period from (A) a pair-fed control rat and (B) a vitamin $\mathrm{D}$-deficient rat. The arrows indicate the inner and outer borders of the zone of initial mineralization. The osteoid which lies superficial to the mineralization front is not fluorescent and appears dark. $\times 425$.

when two or more labels were present at the endosteum. A sequence of two adjacent tetracycline or two adjacent alizarin red $\mathrm{S}$ labels, as could have been expected if cycles of formation and resorption had occurred, never was found. Tetracycline-labeled endosteal bone always was found to be covered by osteoid in rats sacrificed $24 \mathrm{hr}$ after an injection of tetracycline, and neither Howship's lacunae nor intense acid phosphatase activity ever was found along the surface of tetracycline-labeled endosteal bone in rats sacrificed at 2 day intervals throughout the 10 day measuring period. Thus, we found no evidence for resorption of endosteal bone formed during this period.

Endosteal matrix-forming surfaces could be identified without difficulty because bone beneath them was labeled with tetracycline. Although Howship's lacunae occupied a large portion of the remaining unlabeled endosteal surface, they were not always detectable over the entire unlabeled endosteal surface; therefore, we could not rule out the possibility that some of the unlabeled surface was neither forming nor resorbing. Therefore, we simultaneously evaluated matrix-forming and bone-resorbing surfaces at the endosteum using the location of tetracycline deposited in bone after an intraperitoneal injection to identify forming surfaces and extracellular acid phosphatase activity on the endosteal bone surface to identify resorption. We found that all unlabeled endosteal surface displayed an intense extracellular reaction for acid phosphatase, including all of those areas that could be identified as Howship's lacunae. Thus, in these rapidly growing rats, the entire endosteal surface in the mid-tibial diaphysis was involved in either formation or resorption.

The major differences between the two control groups of rats in any of the variables measured were body weight and bone resorption (Tables II and III). The latter may 
TABLE II

Vitamin D Intake and Growth and Serum Values

\begin{tabular}{|c|c|c|c|c|c|c|}
\hline & \multicolumn{2}{|c|}{ Ad lib.-fed control groups } & \multicolumn{2}{|c|}{ Pair-fed control groups } & \multicolumn{2}{|c|}{ Vitamin D-deficient groups } \\
\hline & Basal & Final & Basal & Final & Basal & Final \\
\hline Total vitamin $\mathrm{D}$ intake, $I . U$. & $172^{*}$ & 419 & 185 & 527 & 0 & 0 \\
\hline Body weight, $g$ & $96 \pm 7 \ddagger$ & $156 \pm 12$ & $94 \pm 3$ & $133 \pm 5$ & $91 \pm 7$ & $134 \pm 16$ \\
\hline Tibial length, $\mathrm{mm}$ & & $30.3 \pm 0.7$ & & $29.8 \pm 0.6$ & & $28.9 \pm 1.1$ \\
\hline Serum calcium, $m g / 100 m l$ & $11.0 \pm 0.5$ & $11.0 \pm 0.3$ & $10.9 \pm 0.4$ & $11.1 \pm 0.4$ & $8.5 \pm 1.4$ & $6.6 \pm 1.2$ \\
\hline Serum phosphorus, $m g / 100 \mathrm{ml}$ & $11.2 \pm 0.8$ & $10.3 \pm 1.0$ & $11.3 \pm 1.0$ & $10.3 \pm 0.7$ & $10.9 \pm 0.6$ & $11.0 \pm 0.8$ \\
\hline Serum $\mathrm{Ca} \times \mathrm{P}$ product & $123 \pm 10$ & $114 \pm 11$ & $126 \pm 11$ & $114 \pm 11$ & $93 \pm 17$ & $73 \pm 13$ \\
\hline
\end{tabular}

* Vitamin D intake is calculated from the measured intake of food and the concentration of vitamin D in the diet. The figures given are group means for the total intake during the experimental periods.

$\sharp$ Mean \pm SD. All measurements were made at the time of sacrifice.

TABLE III

Formation, Mineralization, and Resorption of Bone

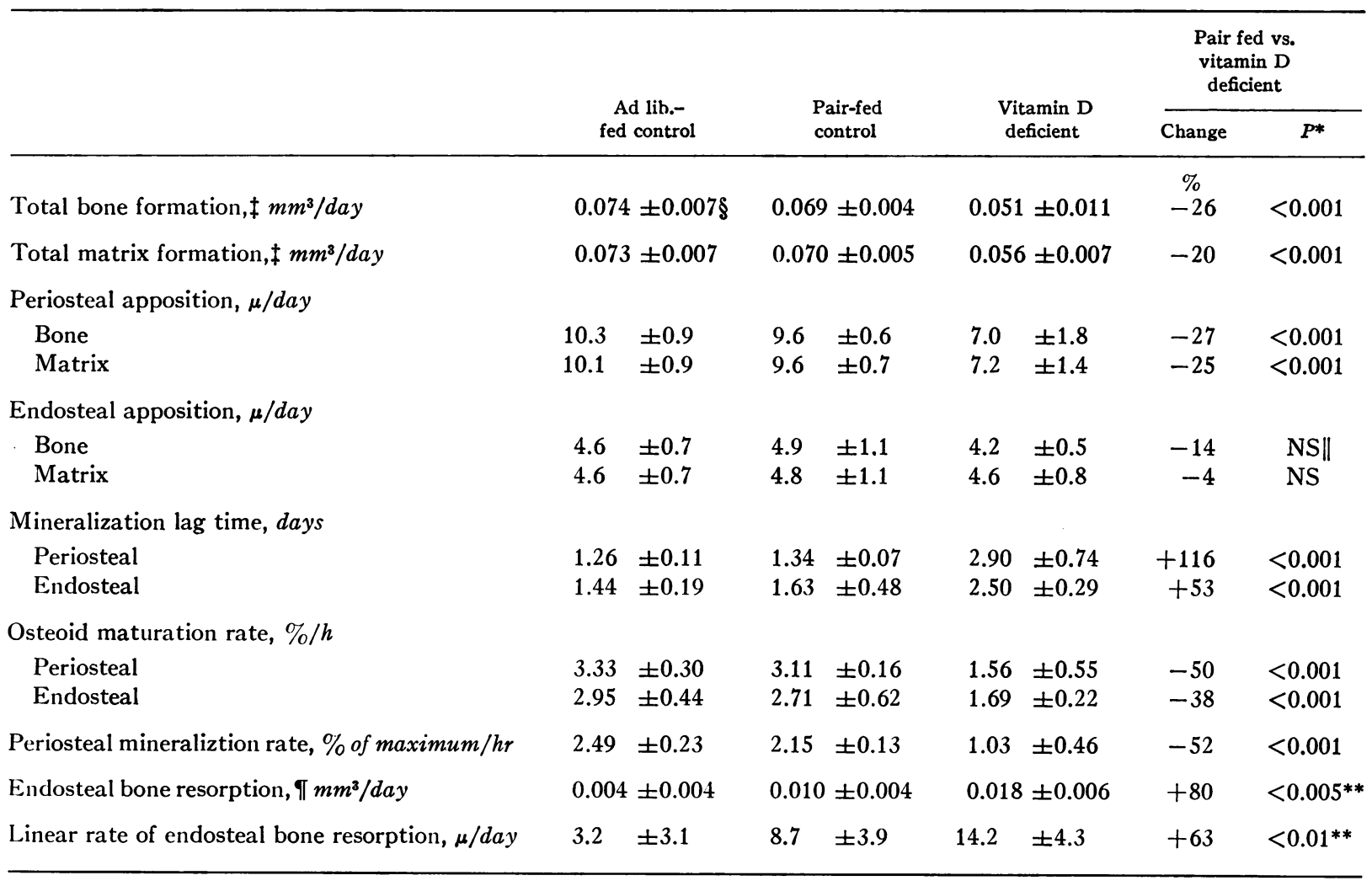

* Probability estimated by $t$ test.

$\ddagger$ Total indicates both periosteal and endosteal formation.

$\S$ Mean \pm SD.

\| Not statistically significant at the 0.05 probability level.

I Represents total resorption, since no resorption occurred at the periosteum.

** Based on variances estimated from the ranking procedure. 
TABLE IV

Correlation Coefficients in the Vitamin D-Deficient Group*

\begin{tabular}{|c|c|c|c|c|c|}
\hline & \multicolumn{3}{|c|}{ Correlation coefficients } & \multicolumn{2}{|c|}{ Partial correlation coefficients } \\
\hline & $\begin{array}{l}\text { Serum } \\
\text { calcium }\end{array}$ & $\begin{array}{c}\text { Serum } \\
\text { phosphorus }\end{array}$ & $\begin{array}{c}\text { Serum } \\
\mathrm{Ca} \times \mathrm{P}\end{array}$ & $\begin{array}{l}\text { Serum } \\
\text { calcium }\end{array}$ & $\begin{array}{c}\text { Serum } \\
\text { phosphorus }\end{array}$ \\
\hline Linear matrix formation rate & $\begin{aligned} r & =0.90 \\
P & <0.001\end{aligned}$ & $\begin{array}{c}r=-0.12 \\
N S \ddagger\end{array}$ & $\begin{array}{c}r=0.82 \\
P<0.005\end{array}$ & $\begin{array}{c}r=0.88 \\
P<0.005\end{array}$ & $\begin{array}{c}r=0.17 \\
\text { NS }\end{array}$ \\
\hline Mineralization lag time & $\begin{array}{l}r=-0.87 \\
P<0.001\end{array}$ & $\begin{array}{c}r=0.01 \\
\text { NS }\end{array}$ & $\begin{array}{l}r=-0.85 \\
P<0.005\end{array}$ & $\begin{array}{l}r=-0.87 \\
P<0.005\end{array}$ & $\begin{array}{c}r=-0.27 \\
\mathrm{NS}\end{array}$ \\
\hline Mineralization rate & $\begin{aligned} r & =0.93 \\
P & <0.001\end{aligned}$ & $\begin{array}{c}r=-0.01 \\
\mathrm{NS}\end{array}$ & $\begin{array}{c}r=0.87 \\
P<0.005\end{array}$ & $\begin{array}{c}r=0.92 \\
P<0.001\end{array}$ & $\begin{array}{c}r=0.15 \\
\text { NS }\end{array}$ \\
\hline
\end{tabular}

* The bone and serum data used were the means of basal and final measurements and thus were all representative of the 10 day measuring period; the bone data used were from periosteal measurements.

$\ddagger$ Not statistically significant at the $\mathbf{0 . 0 5}$ probability level.

have resulted from decreased calcium intake as a consequence of reduced food intake by the pair-fed control group. Body weight and rate of weight gain in the pairfed control and vitamin D-deficient group were the same (Table II) and, therefore, any difference between these two groups represents the effect of vitamin D-deficiency per se or one of its complications other than decreased weight gain or food consumption.

The rates of bone formation, mineralization, and resorption for the vitamin D-deficient and control groups are given in Table III. As compared to the pair-fed control group, the rates of matrix and bone formation were 22 and $26 \%$ less in the vitamin D-deficient group. In our sampling site, only about $20 \%$ of the total matrix formation occurs at the endosteum and about $10 \%$ along vascular canal surfaces (10). Therefore, periosteal matrix formation is the main determinant of the total matrix formation rate. The periosteal matrix apposition rate was $25 \%$ less in the vitamin D-deficient than in the control group (Table III).

The time which elapses between formation of matrix and initiation of mineralization in matrix was found to be relatively consistent in any given group of rats, irrespective of the apposition rate. Thus, there was a relatively wide band of osteoid at the periosteum where matrix apposition was rapid and a narrow band at the endosteum where apposition was slower (Tables I and II and Fig. 3). However, in both locations, the mineralization lag time was about the same (Table III). A comparable similarity of mineralization lag time was found irrespective of the apposition rate and osteoid width in vitamin D-deficient rats (Tables I and II). The periosteal mineralization lag time was twice as long and the osteoid maturation rate was half as rapid in vitamin D-deficient rats as it was in pair-fed control rats (Table III).

The maximum mineral concentration in the minerali- zation front was estimated from refractive index (nD) measurements. In both control and vitamin D-deficient rats, the $\mathrm{nD}$ of osteoid was 1.533 , the maximum $\mathrm{nD}$ of the mineralization front was 1.536 , and the maximum $\mathrm{nD}$ in mature bone was 1.548 . The maximum mineral concentration in the mineralizing front in all three groups, calculated from these data, was $20 \%$ of the maximum mineral concentration in mature bone. The width of the mineralization front was $3.8 \pm 0.6 \mu$ in control and $5.5 \pm 1.7 \mu$ in vitamin $\mathrm{D}$-deficient rats $(\mathrm{P}<0.001)$. The rates of initial mineralization were calculated from these data. This rate was altered to the same degree as was the osteoid maturation rate, being half as rapid in the vitamin D-deficient group than in the control group (Table III).

Table IV gives a correlation matrix for the vitamin D-deficient group, with the concentrations of calcium and phosphorus in serum and the concentration product of calcium and phosphorus ions in serum as the independent variables and matrix apposition rate, mineralization lag time, and initial mineralization rate as the dependent variables. There were strong correlations between these latter variables and the degree of hypocalcemia. The correlations with the ion concentration product of calcium and phosphate in serum was significant but somewhat weaker.

The bone resorption rate was $80 \%$ greater in vitamin D-deficient than in pair-fed control rats. This increase was caused by a large increase in the linear rate of resorption. The lengths of resorbing surfaces were not significantly different statistically in either the basal or final groups of vitamin D-deficient as compared with the pair-fed control rats. When we used analysis of variance and Scheffe's multiple comparison technique (14), the endosteal bone resorption rate was found to be significantly $(P<0.025)$ greater in the vitamin 
D-deficient than in the group of rats fed an unrestricted control diet, but the difference between the vitamin $D$-deficient and pair-fed control group was not statistically significant $(P<0.15)$. However, when we applied the ranking procedure, both the endosteal bone resorption rate and the rate of resorption per unit length of resorption surface were found to be significantly greater in the vitamin D-deficient than in the pair-fed control group (Table III).

\section{DISCUSSION}

The total rate of bone formation by osteoblasts depends upon two processes, the extent of the surface of bone along which osteoblasts actively form bone (forming surface) and the volume of bone formed per unit area of forming surface per unit of time (apposition rate). Similarly, osteoclastic bone resorption is a function of two processes, the extent of surface of bone along which osteoclasts resorb bone (resorbing surface) and the volume of bone resorbed per unit of time per unit area of resorbing surface. This latter is analogous to the apposition rate and is termed by us the linear resorption rate. Each of these variables can be interpreted in terms of more fundamental characteristics of bone; the forming and resorbing surfaces are related to the number of osteoblasts and osteoclasts, while the apposition rate and linear resorption rate are related to the rates of matrix formation or bone resorption per osteoblast or osteoclast.

Method. The method for measurement of bone resorption is based upon the principal advanced by $\mathrm{Wu}$, Jett, and Frost to estimate resorption in a section of human rib (18). However, in our work, all of the assumptions necessary for the calculation have been validated. Furthermore, by use of acid phosphatase staining, we have been able to determine the entire extent of surfaces undergoing osteoclastic resorption. Thus, the method we have developed measures for the first time the total bone resorption rate and the linear rate of bone resorption. The precision of the method has been enhanced by a number of technical procedures, including the following. (a) The site from which the sample is taken can be identified precisely, the sections were taken perpendicular to the long axis of the bone, and the processes at the entire periosteal and endosteal borders were evaluated. Together, these eliminated or minimized errors due to sample variability and obliquity of the sample examined. (b) The measurement of bone formation was corrected for uptake of tetracycline at the mineralization front. If this correction had not been made, the amount of bone formed in the vitamin D-deficient group would have been overestimated because of the increased width of the mineralization front in this group.
Several limitations of this method also deserve comment. (a) These measurements were made in growing rats and therefore the data are not necessarily applicable to bone remodeling in adult rats or human beings. (b) Our measurements are confined to the diaphyses and possibly might not be representative of the entire skeleton. However, the most reasonable assumption is that changes of these rates would be similar throughout the skeleton. (c) Since we cannot measure the area of the medullary cavity at the start of the labeling period of those bones destined for measurement of medullary cavity area at the end of that period, we had to measure medullary area in a separate group of rats sacrificed at the beginning of the labeling period for our calculation of bone resorption. Although this in no way alters the accuracy of the group-mean values of resorption so calculated, it does increase the variance. ( $d$ ) In rats during the period between 33 and 43 days of age, there are substantial reductions in the rates of bone formation and resorption. Therefore, the mean rates calculated from measurements made over a period of 10 days are not representative of each day during that period. (e) The site we use in the tibial diaphysis might not be optimal for observing a change in the lengths of formation and resorption surfaces because in this site in growing rats all surfaces are involved either in formation or resorption. Therefore, a change in one type of surface activity must be accompanied by a reciprocal change in the other type of surface activity. Despite this limitation, a marked increase in resorbing surface was obtained when this method was used to study calcium deficient rats."

Vitamin $D$ deficiency. The major bone changes found as a result of vitamin D deficiency were $(a)$ decreased osteoblastic matrix formation, $(b)$ inhibition of mineralization, and (c) increased osteoclastic bone resorption (Table III).

Despite comparable weight gains, the rates of osteoblastic matrix formation and bone formation were significantly less in the vitamin D-deficient than in control rats. The observed reduction in total matrix formation can be accounted for entirely on the basis of reduced apposition; this is illustrated by the findings that the rate of periosteal matrix apposition was decreased by $25 \%$, whereas the periosteal matrix-forming surface was not significantly different (Tables I and III). These results are consistent with histological findings (3) and measurements of proline- ${ }^{14} \mathrm{C}$ incorporation into bone matrix (2) which also show reduced bone synthesis in vitamin $\mathrm{D}$ deficiency. The mineralization of lag time and the osteoid maturation rate have not previously been measured and deserve comment. In normal animals,

\footnotetext{
'Stauffer, M., D. J. Baylink, J. Wergedal, and C. Rich. Manuscript in preparation.
} 
osteoid widths vary by about $100 \%$ within any given bone section, being wide at the periosteum where apposition is rapid (Table III). However, it is our finding that, in these different locations, the mineralization lag time is nearly the same (Table III). We believe that one or several undefined processes must occur in osteoid between when it is formed and when it is able to mineralize. Accordingly, the mineralization lag time can be viewed as a measure of the time required for these processes. Its reciprocal function, the osteoid maturation rate, is a measurement of these processes. Although the osteoid maturation rate has not previously been determined quantitatively, the concept that there may be such a process is not new. The facts that osteoid fails to become mineralized for some period of time ( 1 day in normal rats and 10 days in normal man), that osteoid differs histochemically from bone matrix, and that histochemical changes occur in osteoid before the initiation of mineralization $(16,19-21)$, have been advanced as evidence that some process of maturation must occur before mineralization is possible $(11,19)$. Presumably, such changes occur because of release of enzymes or other cell products into the matrix $(16,22)$.

All of the processes related to matrix deposition and mineralization that we measured were inhibited in the vitamin D-deficient rats (Tables II and III). Since osteoid maturation was more slowed than matrix apposition, the width of the band of osteoid was greater than normal in the vitamin D-deficient rats. The osteoid maturation rate and the rate of initial mineralization both were about $\frac{1}{2}$ of normal. The fact that both these rates were altered to the same degree suggests that both the maturation of osteoid and the rate of initial mineralization reflect different phases in a single over-all process, with the observed changes of vitamin D deficiency resulting from some interference with the metabolism of osteoblasts and young osteocytes.

The correlations between measurements of bone formation and mineralization and the concentration of calcium in serum of the vitamin D-deficient rats all were slightly stronger than those with the ion concentration products of serum calcium and phosphate. This appeared to be because the latter included a function of an uncorrelated variable, the serum phosphate concentration ( $\mathrm{Ta}$ ble IV). These correlations fail to exclude the possibility that the product of the calcium and phosphate ion concentrations may govern the rate of osteoid maturation or the rate of initial mineralization or both, but are more favorable to several other possibilities: $(a)$ that inhibition of bone formation and hypocalcemia each are proportional to the degree of vitamin D deficiency, $(b)$ that the inhibition of bone formation is caused by hypocalcemia, and (c) that this inhibition is caused by some variable which is proportional to the degree of hypo- calcemia, such as secondary hyperparathyroidism. Because we ${ }^{7}$ and others (23) find inhibition of formation and mineralization of matrix in hypocalcemic animals which are not vitamin $\mathrm{D}$ deficient, we believe one of the last two of these possibilities is more likely than the first.

The reduced rate of bone formation and increased osteoclastic bone resorption observed in vitamin $\mathrm{D}$ deficiency would both be expected to promote hypercalcemia rather than hypocalcemia. Despite this, the animals developed hypocalcemia. Obviously, bone resorption, although greater than in the control animals, was not increased to the degree necessary to compensate for the deficiency-induced reduction of absorption of calcium from the intestine. However, vitamin D-deficient rats continue to absorb an appreciable fraction of the dietary calcium and these animals probably received internally about half of the amount of calcium once they had become vitamin $\mathrm{D}$ deficient than they would have otherwise (24). Vitamin D-treated rats on a diet which contains no calcium do not become as hypocalcemic as vitamin D-deficient rats, presumably because increased bone resorption nearly compensates for complete lack of calcium in the diet. " Thus, in vitamin D-deficient rats, as compared to normal animals, resorption is increased, but far less so than is found in vitamin D-fed calcium-deficient animals which had less marked hypocalcemia. Because of these considerations, we conclude that there is a defect of osteoclastic bone resorption in vitamin $\mathrm{D}$ deficiency. This conclusion is consistent with results of other studies all pointing towards a resistance to the action of parathyroid hormone in vitamin D-deficient animals $(25,26)$. Since we found very little increase in the osteoclastic resorbing surface, it would appear that, in the absence of vitamin $\mathrm{D}$, the differentiation of osteoclasts may be inhibited to a much greater degree than the resorptive activity per osteoclast. Finally, to the extent that osteocytes participate in calcium homeostasis $(27,28)$, the same considerations would suggest that they must also be similarly resistant to parathyroid hormone in vitamin $\mathrm{D}$ deficiency.

Our present concept, which we consider most consistent with our results and with unpublished data, ${ }^{7}$ is that the primary effect of vitamin $\mathrm{D}$ deficiency on bone is a reduction in the capacity of osteoclastic and probably of osteocytic resorption. This, along with decreased intestinal absorption of calcium, promotes hypocalcemia and secondary hyperparathyroidism. Bone cells presumably can respond only suboptimally to the increased concentration of parathyroid hormone in blood and the response becomes less effective as the degree of vitamin $\mathrm{D}$ deficiency becomes more profound. Accordingly, hypocalcemia becomes more severe even though the concentration of parathyroid hormone in blood presumably 
increases. Finally our data, combined with those of others, are consistent with the possibility that hypocalcemia or secondary hyperparathyroidism rather than vitamin $\mathrm{D}$ deficiency per se causes the reduced rates of formation and mineralization of bone matrix.

\section{ACKNOWLEDGMENTS}

We are grateful to Dr. M. Leyton, Department of Preventive Medicine, University of Washington, for assistance in statistical analyses; Mr. M. Takamoto, Mr. K. Douglass, Mr. G. Perez, Mr. R. Haller, and Mr. H. Fellows for their invaluable technical assistance; Mr. D. Parrish and Mrs. K. Hashimoto for computer assistance; and Mrs. B. Herold for typing the manuscript.

This study was supported in part by Grant AM-9096 from the U. S. Public Health Service.

\section{REFERENCES}

1. Fraser, R., M. Harrison, and K. Ibbertson. 1960. The rate of calcium turnover in bone. Measurement by a tracer test using stable strontium. Quart. J. Med. 29: 85.

2. Canas, F., J. S. Brand, W. F. Neuman, and A. R. Terepka. 1969. Some effects of vitamin $D_{3}$ on collagen synthesis in rachitic chick cortical bone. Amer. J. Physiol. 216: 1092.

3. Kelly, P. J. 1967. Bone remodeling in puppies with experimental rickets. J. Lab. Clin. Med. 70: 94.

4. Aubert, J. P., and B. Milhaud. 1960. Method for the measurement of the principal routes of calcium metabolism in man. Biochim. Biophys. Acta. 39: 122.

5. Wergedal, J. 1969. Enzymes of protein and phosphate catabolism in rat bone. I. Enzyme properties in normal rats. Calcified Tissue Res. 3: 55 .

6. Willis, J. B. 1960. The determination of metals in blood serum by atomic absorption spectroscopy-I. Calcium. Spectrochim. Acta. 16: 259

7. Fiske, C. H., and H. Y. Subbarow. 1925. The colorimetric determination of phosphorus. J. Biol. Chem. 66: 375.

8. Heller-Steinberg, M. 1951. Ground substance, bone salts and cellular activity in bone formation and destruction. Amer. J. Anat. 89: 347.

9. Herzberg, K. 1934. 7 Victoriablau zur Farbung von filtreierborem viris. Zentrabl. Bakteriol. Parasitenk. Infektionshkr. Hyg. Abt. Orig. 13: 258.

10. Baylink, D., E. Morey, and C. Rich. 1969. Effect of calcitonin on the rates of bone formation and resorption in the rat. Endocrinology. 84: 261.

11. Frost, H. M. 1963. Bone Remodeling Dynamics. Charles C Thomas, Springfield, Ill.

12. Ascenzi, A., and C. Fabry. 1959. Technique for dis- section and measurement of refractive index of osteones. J. Biophys. Biochem. Cytol. 6: 139.

13. Allen, R. M. 1962. Practical refractometry by means of the microscope. R. P. Cargille Labs., Inc. Cedar Grove, N. Y.

14. Cochran, W. G., and G. M. Cox. 1957. Experimental Design. John Wiley \& Son, New York. 2nd edition.

15. Burstone, M. S. 1958. Histochemical demonstration of acid phosphatases with naphthol AS-phosphates. J. Nat. Cancer Inst. 21: 523.

16. Wergedal, J., and D. Baylink. 1969. Distribution of acid and alkaline phosphatase activity in undemineralized sections of the rat tibial diaphysis. J. Histochem. Cytochem. 17: 799.

17. Handelman, C. S., A. Morse, and J. T. Irving. 1964. The enzyme histochemistry of the osteoclasts of normal and "ia" rats. Amer. J. Anat. 115: 363.

18. Wu, K., S. Jett, and H. M. Frost. 1967. Bone resorption rates in rib in physiological, senile, and postmenopausal osteoporoses. J. Lab. Clin. Med. 69: 810.

19. Löe, H. 1959. Bone tissue formation: a morphological and histochemical study. Acta Odontol. Scand. 17: 311.

20. Irving, J. T. 1959. A histological staining method for sites of calcification in teeth and bone. Arch. Oral Biol. 1: 89 .

21. Baylink, D. J., and D. S. Bernstein. 1967. The effects of fluoride therapy on metabolic bone disease. Clin. Orthop. Related Res. 55: 51.

22. Nichols, G., Jr., B. Flanagan, and J. van der Sluys Veer. 1969. Distortions of bone cell metabolism in uremia and their cause. Arch. Intern. Med. 124: 530.

23. Burkhart, J. M., and J. Jowsey. 1966. Morphologic evidence of osteomalacia in the parathyroidectomized dog. Mayo Clin. Proc. 41: 663.

24. Stauffer, M., and C. Rich. 1967. The direct action of vitamin D and lactose on bone in osteomalacia. J. Clin. Invest. 46: 1119.

25. Au, W. Y. W., and F. C. Bartter. 1966. Effect of vitamin $\mathrm{D}$ on in vitro bone calcium metabolism. Endocrinology. 78: 1100 .

26. Rasmussen, H., H. DeLuca, C. Arnaud, C. Hawker, and Marit von Stedingk. 1963. The relationship between vitamin $\mathrm{D}$ and parathyroid hormone. J. Clin. Invest. 42: 1940.

27. Belanger, L. F., J. Robichon, B. B. Migicovsky, D. H. Copp, and J. Vincent. 1963. Resorption without osteoclasts (osteolysis). In Symposium on Mechanism of Hard Tissue Destruction. R. F. Sognnaes, editor. Amer. Ass. Advan. Sci. Publ. 531.

28. Baylink, D. J., E. Morey, and C. Rich. 1968. Effect of calcitonin on osteocyte mineral transfer in the rat. In Parathyroid Hormone and Thyrocalcitonin (Calcitonin). R. V. Talmage, L. F. Belanger and I. Clark, editors. Excerpta Medica Foundation, New York. 196. 